\title{
Heartburn and other related symptoms are independent of body mass index in irritable bowel syndrome
}

\author{
M. Schmulson ${ }^{1}$, D. Pulido ${ }^{1}$, C. Escobar ${ }^{1}$, B. Farfán-Labone ${ }^{1}$, G. Gutiérrez-Reyes ${ }^{1}$ and J. C. López- \\ Alvarenga ${ }^{2}$ \\ 'Laboratory of Liver, Pancreas and Motility (HIPAM). Department of Experimental Medicine-Faculty of Medicine. \\ Universidad Nacional Autónoma de México (UNAM). ${ }^{2}$ Core of Biostatistics. Department of Clinical Research. Hospital \\ General de México, and Department of Genetics. Southwest Foundation for Biomedical Research. San Antonio, Texas. \\ USA
}

\begin{abstract}
Background: increasing body mass index (BMI) is a risk factor for GERD but little is known about this association in the irritable bowel syndrome (IBS).

Aims: to determine the presence of heartburn and other related symptoms in relation with BMI in IBS.

Methods: volunteers $(n=483)$ answered the Rome II-Modular Questionnaire, and were divided into IBS and non-IBS (controls) groups. The frequency of heartburn, chest pain, epigastric pain, nausea, vomiting and belching was compared between the groups in the study sample and within three BMI categories.

Results: the IBS (23.7\%) and controls (76.3\%) were similar in gender (females: 68.1\%), age (32.2 \pm 12.7 years), and BMI (25.4 \pm 4.4). Raw associations analysis showed that heartburn: OR: 1.62 (95\%CI: 1.04-2.53), chest pain: 1.77 (1.13-2.77), epigastric pain: 1.75 (1.03-2.98) and nausea: 2.45 (1.10-5.32) were more frequent in IBS vs. controls. Meanwhile, according to BMI, in those with obesity, heartburn was more frequent in IBS and among those with overweight, epigastric pain and nausea were also more frequent in IBS. However, in an adjusted log linear model, no significant interaction was found between BMI and any other studied symptom and heartburn was found to be independent of IBS: $1,4(0.9,4.7)$. Finally, a logistic regression model found no interaction between $\mathrm{BMI}$ and the presence of heartburn or IBS.
\end{abstract}

Conclusions: while heartburn and other reflux-related symptoms are more frequent in IBS than in controls, these associations are independent of BMI.

Key words: Irritable bowel syndrome (IBS)/epidemiology/diagnosis. Rome II criteria. Body mass index. Heartburn. Dyspepsia. Nausea. Mexico.

\section{Received: 08-10-09.}

Accepted: 30-11-09.

Correspondence: Max Schmulson W. Laboratorio de Hígado, Páncreas y Motilidad (HIPAM). Departamento de Medicina Experimental. Facultad de Medicina-Universidad Nacional Autónoma de México (UNAM). Hospital General de México. Doctor Balmis \#148. Col. Doctores C.P. 06726. México D.F. México.e-mail: maxjulio@prodigy.net.mx
Schmulson M, Pulido D, Escobar C, Farfán-Labone B, Gutiérrez-Reyes G, López-Alvarenga JC. Heartburn and other related symptoms are independent of body mass index in irritable bowel syndrome. Rev Esp Enferm Dig 2010; 102: 229-233.

\section{INTRODUCTION}

Patients with irritable bowel syndrome (IBS) tend to have extraintestinal manifestations such as gastroesophageal reflux disease (GERD), and the evidence has shown an overlap between both disorders. In fact, a study reported that reflux symptoms were the most powerful predictors of extraintestinal manifestations in IBS (1). Furthermore, a recent systematic review of the literature found that the maximum mean prevalence of GERD in patients already diagnosed with IBS was $39 \%$ and the maximum mean prevalence of IBS in subjects with known GERD was 49\%, exceeding the individual presence of each condition (2). Also, in a study aimed to assess the effect of IBS on the postoperative outcome of laparoscopic fundoplication, surprisingly, $81 \%$ of the GERD patients with IBS according to the Rome II criteria, no longer fulfilled criteria for IBS (3). Although controversial, the above mentioned finding suggests that GERD treatment may have a positive impact on the IBS symptoms.

GERD includes a diversity of patients such as those with esophageal erosions and Barrett's esophagus and those with classic symptoms in the presence of a normal esophageal mucosa, considered to have non-erosive reflux disease (NERD), also called endoscopy negative reflux disease (4). Among the symptoms, heartburn is highly specific of GERD, however, an important subgroup of NERD patients known as functional heartburn have esophageal acid exposure within the physiological range in addition to normal endoscopy (4). According to the Rome committee, functional heartburn is defined as an 
episodic retrosternal burning in the absence of pathological gastro-esophageal reflux, motility disorders or structural explanation (5).

On the other hand, GERD has been shown to be more common in obese patients than in those with a body mass index (BMI) within normal range and an increase in the BMI above the 95 percentile for age and gender, is a significant risk factor for GERD $(6,7)$. Also, a higher BMI is associated with more frequent and more severe heartburn and regurgitation in patients with GERD and an increasing BMI is a strong predictor of heartburn during sleep $(8,9)$.

It is suggested that there is a common link between IBS and GERD; however, there is no convincing evidence for a common underlying pathophysiology or somatization (10). Considering that an increasing BMI is a risk factor for GERD, we hypothesized that the presence of heartburn and other related symptoms among subjects with IBS, is related to an increasing BMI. Therefore, we sought to explore whether the presence of heartburn and other reflux-related symptoms according to the Rome II Modular Questionnaire, was related to BMI in IBS subjects from the community.

\section{METHODS}

Volunteers from a university-based population that were invited by advertisement to participate in an epidemiological study for functional gastrointestinal disorders in Mexico City were included. After signing an informed consent, they all answered the Rome II Modular Questionnaire in Spanish-Mexico $(11,12)$.

Subjects were classified as IBS and non-IBS (controls). IBS was diagnosed if subjects had often (symptoms were present during at least 3 weeks -at least one day in each week- in the last 3 months) abdominal discomfort or pain with two of the following criteria: relief after a bowel movement and/or; it is associated with a change in the frequency of the bowel movements; and/or it is associated with a change in the consistency of the bowel movements. IBS was further classified based on the bowel habit predominance as diarrhea (IBS-D), constipation (IBS-C), and alternating (IBS-A) (11).

The frequency during the previous 3 months of heartburn and other related symptoms included in the Rome II Modular Questionnaire $(11,12)$, were analyzed. Accordingly, heartburn was considered in the presence of a burning pain or discomfort in the chest at least once a week in the absence of any difficulty after swallowing. Chest pain was determined in the presence of pain in the middle of the chest, not due to angina or a heart attack. Also, patients were asked about discomfort or pain centered in the upper abdomen, above the belly button, or in the pit of the stomach. Those answering yes to the prior question, were asked to better classify their symptom as pain (considered in our study as epigastric pain) or discomfort. The latter ones further characterized their discomfort to determine the presence of nausea. Vomiting was considered in the presence of episodes on at least 3 separate days in each week, and finally the presence of burp or belch (belching) was determined.

Weight and height were measured in all subjects and the BMI was calculated as weight in kilograms divided by the square of height in meters $\left(\mathrm{kg} / \mathrm{m}^{2}\right)$ and further categorized as follows: underweight in those with a BMI < 18.50, normal range in 18.50-24.99, overweight in $\geq 25$ and obesity in $\geq 30$. Obesity was further divided as class I (BMI: $\geq 30$ to $<35$ ), class II (BMI: $\geq 35$ to $<40$ ) and class III $(\geq 40)(13)$.

\section{Statistical analysis}

Continuous variables are expressed in mean $\pm \mathrm{SE}$ and were analyzed by the Student's t test and categorical variables are expressed in percentages and were analyzed by the $\chi^{2}$. Symptom comparisons between IBS and controls were analyzed by the Fisher's exact test and results are expressed as odds ratio (OR) and 95\% confidence intervals $(95 \% \mathrm{CI})$. A log linear analysis was run to search for interactions between the studied variables (IBS, heartburn, chest pain, epigastric pain, nausea, vomiting, belching, BMI, age, gender). Selection with backwards elimination process was used to assess for significant interactions $(p<0.05)$ in groups of three variables followed by the search of significant interactions in groups of two variables. The significant interactions were introduced in a Poisson model to determine association coefficients. Also, a logistic regression analysis with backwards stepwise elimination process was run to determine the factors associated with IBS and heartburn using the BMI categories as the variable of interest. The model included ordinal variables (as main effects) to increase the statistical efficiency of the logistic regression. We included IBS, heartburn, the IBS*heartburn interaction, BMI (normal, overweight, obesity), age (quartiles), gender as independent variables, while chest pain, epigastric pain, nausea, vomiting and belching, as dependent variables. To be included as a significant interaction in the model, a $\mathrm{p}<0.10$ was necessary.

\section{RESULTS}

\section{Subjects demographics}

A total of 483 volunteers were studied. Of them, 113 (23.4\%) fulfilled criteria for IBS and 370 (76.0\%) were controls. Both groups were similar in gender distribution, age, marital status, weight, height and BMI (Table I).

Subjects distribution according to BMI categories, included (IBS vs. controls) underweight: $0 v s .2 .4 \%$; normal: 50.4 vs. $50.7 \%$; overweight: 39.1 vs. $32.6 \%$; obesity I: 7.0 
Table I. Demographic characteristics in IBS and controls

\begin{tabular}{llccc}
\hline & & $\begin{array}{c}\text { IBS } \\
(n=113)\end{array}$ & $\begin{array}{c}\text { Controls } \\
(n=370)\end{array}$ & $p$ \\
\hline Gender & F/M frequency & $77 / 36$ & $252 / 118$ & 0.995 \\
\hline Age & Years: mean \pm SE & $31.5 \pm 1.3$ & $32.3 \pm 0.7$ & $0.549^{*}$ \\
\hline Age quartiles & $\leq 21$ years: $n(\%)$ & $29(25.7)$ & $90(24.3)$ & 0.881 \\
& $22-27$ years: $n(\%)$ & $31(27.4)$ & $91(24.6)$ & \\
& 28 -42 years: $n(\%)$ & $29(25.7)$ & $101(27.3)$ & \\
& $\geq$ 43 years: $n(\%)$ & $24(21.2)$ & $88(23.8)$ & \\
\hline Marital status $(\%)$ & Single (\%) & $71(62.8)$ & $222(59.9)$ & 0.885 \\
& Married (\%) & $28(24.8)$ & $112(30.3)$ & \\
& Civil union (\%) & $5(4.4)$ & $13(3.4)$ & \\
& Separated/divorced (\%) & $7(6.2)$ & $19(5.2)$ & \\
& Widow/widower (\%) & $2(1.8)$ & $4(1.2)$ & \\
\hline BMl & Mean \pm SE & $25.3 \pm 0.4$ & $25.5 \pm 4.5$ & $0.737^{*}$ \\
\hline BMl categories & Normal: $n(\%)$ & $56(49.6)$ & $196(53.0)$ & 0.308 \\
& Overweight: $n(\%)$ & $45(39.8)$ & $121(32.7)$ & \\
& Obesity: $n(\%)$ & $12(10.6)$ & $53(14.3)$ & \\
\hline
\end{tabular}

The table depicts the demographic characteristics in IBS and controls. The groups were similar in gender, age, marital status, weight, height and BMI. *Contrast analyzed with the Student's t test, other associations were tested with the Chisquare.

vs. $10.5 \%$; obesity II: 3.5 vs. $3.2 \%$; obesity III: 0 vs. $0.6 \%$; p $=0.340$. In order to increase the statistical power, subjects with underweight were included in the normal category and the three classes of obesity were grouped in one category (obesity). Accordingly, neither were there any differences in the frequency of these BMI categories between the groups (IBS vs. controls), normal: $50.4 v s .53 .1 \%$; overweight: 39.1 vs. $32.6 \%$; and obesity: 10.5 vs. $14.3 \%$; $\mathrm{p}=0.336$. The bowel habit subgroups among the IBS subjects were, IBS-D: 35.7\%; IBS-C: $19.1 \%$; IBS-A: $45.2 \%$; and there were no differences in BMI among them (data not shown).

\section{Heartburn and other related symptoms in IBS and controls}

The frequency of heartburn, chest pain, epigastric pain, nausea, vomiting and belching, is shown in table II. Except for vomiting and belching, they were all significantly more frequent in IBS compared to controls (Table II).

Specifically, regarding the relationship of heartburn with IBS, the frequency of this overlap was $(47.9 \%)$ higher than the individual prevalence of IBS: $23.7 \%$, OR (95\% CI): $3.17(2.03-4.93), \mathrm{p}<0.001$ and a trend over the individual presence of heartburn itself: $40.5 \%$, OR (95\%CI): $1.44(0.94-2.13), \mathrm{p}=0.093$.

\section{Sample stratified according to BMI}

When the study population was stratified according to the analyzed BMI categories, in the subjects with obesity,
Table II. Frequency of heartburn and other reflux related symptoms in volunteers with IBS and controls

\begin{tabular}{lccccc}
\hline & $\begin{array}{c}\text { All } \\
(n=483)\end{array}$ & $\begin{array}{c}\text { IBS } \\
(n=113)\end{array}$ & $\begin{array}{c}\text { Controls } \\
(n=370)\end{array}$ & & IBS vs. controls \\
\hline Symptoms & $(\%)$ & $(\%)$ & $(\%)$ & OR (95\% Cl) & $p$ \\
Heartburn & 40.5 & 49.6 & 37.7 & $1.62(1.04-2.53)$ & $0.030^{*}$ \\
Chest pain & 35.0 & 45.2 & 31.8 & $1.77(1.13-2.77)$ & $0.010^{*}$ \\
Epigastric pain & 43.8 & 54.2 & 40.3 & $1.75(1.03-2.98)$ & $0.000^{*}$ \\
Nausea & 12.3 & 20.9 & 9.7 & $2.45(1.10-5.32)$ & $0.003^{*}$ \\
Vomiting & 7.7 & 7.8 & 7.7 & $1.02(0.41-2.30)$ & 0.205 \\
Belching & 46.7 & 54.8 & 44.1 & $1.53(0.99-2.39)$ & 0.054 \\
\hline
\end{tabular}

The table depicts the frequency of heartburn, chest pain, epigastric pain, nausea, vomiting and belching in the study sample (All) as well as in IBS vs. controls. Raw associations analysis showed that all the symptoms were significantly $\left({ }^{*}\right)$ more frequent in IBS vs. controls, except for vomiting and belching.

heartburn was more prevalent in IBS $v s$. controls $(\mathrm{p}=$ $0.024)$ while in those with overweight, epigastric pain ( $\mathrm{p}$ $=0.002)$ and nausea $(\mathrm{p}=0.008)$ were also more frequent in IBS. There were no differences in IBS $v s$. controls in the normal category (Table III). However, in the log linear model of three variables interaction, it was suggested that only chest pain may be related to obesity in the younger group of patients. In the two variables model, there were no interactions between BMI and any symptom, and heartburn was found to be independent of IBS: $1.4(0.9,4.7)$. Only the following symptoms showed significant interactions: nausea and heartburn: $2.7(1.5,4.7)$; chest pain and heartburn: $2.2(1.5,3.3)$; epigastric pain and burping: $1.6(1.5,2.4)$; chest pain and epigastric pain: 3.3 (1.4, 8.0). Furthermore, in the logistic regression analysis no interaction was found between BMI or age, with the presence of heartburn, nor IBS (Table IV).

\section{DISCUSSION}

The overlap between GERD and IBS is well known (1-2) and although they may be two manifestations of similar underlying pathophysiological processes, this remains to be elucidated (9). In the current study we found that heartburn according to the Rome II Modular Questionnaire and other related symptoms such as chest pain, epigastric pain and nausea, were significantly more frequent in IBS than controls among volunteers recruited by advertisement from a university-based population in Mexico. In addition, when the study population was stratified according to the BMI categories, in the subjects with obesity, heartburn was more likely to be present in IBS compared to controls and in those with overweight, epigastric pain and nausea, were also more common in IBS. However, the logistic regression analysis showed that these symptoms associations in IBS were independent of BMI.

In the current study, the frequency of heartburn in IBS was higher than the individual presence of IBS and had a trend over that of heartburn itself. These results are very similar to those of a recent systematic review by Nas- 
Table III. Frequency of heartburn and other related symptoms in IBS and controls, within the different BMI categories

\begin{tabular}{|c|c|c|c|c|c|c|}
\hline & Heartburn & Chest pain & Epigastric pain & Nausea & Vomiting & Belching \\
\hline $\begin{array}{l}\text { BMI categories } \\
\mathrm{N} \text { (IBS; controls) }\end{array}$ & $\begin{array}{c}\text { Frequency }(\%) \text { IBS; controls } \\
\text { OR }(95 \% \mathrm{Cl})\end{array}$ & & & & & \\
\hline $\begin{array}{l}\text { Normal } \\
(56 ; 196)\end{array}$ & $\begin{array}{c}48.3 ; 36.5 \\
0.62(0.33,1.17)\end{array}$ & $\begin{array}{c}48.3 ; 33.5 \\
0.54(0.29,1.02)\end{array}$ & $\begin{array}{c}48.9 ; 47.1 \\
0.93(0.42,1.93)\end{array}$ & $\begin{array}{c}19.0 ; 11.7 \\
0.56(0.24,1.38)\end{array}$ & $\begin{array}{c}8.6 ; 9.2 \\
1.10(0.36,3.89)\end{array}$ & $\begin{array}{c}51.7 ; 42.3 \\
0.68(0.36,1.29)\end{array}$ \\
\hline $\begin{array}{l}\text { Overweight } \\
(45 ; 121)\end{array}$ & $\begin{array}{c}46.7 ; 43.0 \\
0.86(0.41,1.82)\end{array}$ & $\begin{array}{c}42.2 ; 28.1 \\
0.53(0.25,1.17)\end{array}$ & $\begin{array}{c}60.0 ; 30.4 \\
0.30(0.11,0.79)^{*}\end{array}$ & $\begin{array}{c}24.4 ; 8.3 \\
0.28(0.10,0.80)^{*}\end{array}$ & $\begin{array}{c}6.7 ; 6.0 \\
0.90(0.19,5.64)\end{array}$ & $\begin{array}{c}53.3 ; 47.5 \\
0.79(0.37,1.66)\end{array}$ \\
\hline $\begin{array}{l}\text { Obesity } \\
(12 ; 53)\end{array}$ & $\begin{array}{c}66.7 ; 30.2 \\
0.22(0.04,0.09)^{*}\end{array}$ & $\begin{array}{c}41.7 ; 34.0 \\
0.72(0.17,3.32)\end{array}$ & $\begin{array}{c}62.5 ; 36.8 \\
0.35(0.05,2.17)\end{array}$ & $\begin{array}{c}16.7 ; 5.7 \\
0.23(0.03,4.12)\end{array}$ & $\begin{array}{c}8.3 ; 5.8 \\
0.67(0.05,38.5)\end{array}$ & $\begin{array}{c}75.0 ; 43.3 \\
0.26(0.04,1.20)\end{array}$ \\
\hline
\end{tabular}

The table shows the frequency of heartburn and other reflux-related symptoms in IBS and controls according to the BMI categories stratification of the study population The following symptoms were significantly more frequent in IBS vs. controls*: in volunteers with overweight, epigastric pain $(p=0.006)$ and nausea $(p=0.008)$; and heartburn $(p=0.022)$ among those with obesity.

Table IV. Logistic regression analysis for symptoms related with IBS and heartburn

\begin{tabular}{|c|c|c|c|c|c|c|}
\hline Variable & $\begin{array}{l}\text { Heartburn } \\
\text { OR (95\% Cl) }\end{array}$ & Chest pain & Epigastric pain & Nausea & Vomiting & Belching \\
\hline Gender & - & $1.84(1.19,2.84)$ & $2.34(1.47,3.70)$ & $1.92(0.99,3.72)$ & $2.57(1.05,6.29)$ & - \\
\hline Age & - & - & - & - & - & - \\
\hline $\mathrm{BMl}$ & - & - & - & - & - & - \\
\hline IBS & $1.58(1.03,2.41)$ & $1.60(1.02,2.50)$ & $1.74(1.12,2.73)$ & $4.4(1.82,10.62)$ & - & - \\
\hline $\mathrm{BMI} \times \mathrm{IBS}$ & - & - & - & - & - & $1.52(0.99,2.35)$ \\
\hline Heartburn & Not used & $2.40(1.62,3.56)$ & - & $4.0(1.92,8.32)$ & - & $2.25(1.55,3.28)$ \\
\hline Heartburn x IBS & Not used & - & - & $0.30(0.09,0.96)$ & - & - \\
\hline
\end{tabular}

The table shows the results of the logistic regression analysis for the associations between the independent variables (rows) with dependent variables (columns). IBS is the main variable to explain many of the upper abdominal symptoms. The early finding of the BMI effect was confounded by heartburn, IBS and gender. The interaction between heartburn and IBS was significant for the presence of nausea. Each model was set using the backwards stepwise elimination with a criterion of a $p<0.10$.

taskin et al., which analyzed the overlap between IBS and GERD in the general population (2). In our study we only analyzed the presence of heartburn according to the Rome II Modular Questionnaire but we did not rule out pathological gastroesophageal reflux to discriminate those subjects from the ones with functional heartburn, as precluded by the Rome II criteria (5). However, in a recent study in Mexico, we found that more than $60 \%$ of those reporting heartburn according to the Rome II Modular Questionnaire were positive for GERD by $\mathrm{pH}$ monitoring and/or endoscopic examination, suggesting that in our population only one third of these patients have functional heartburn (14). In addition, we did not analyze the onset of the first manifestation of heartburn or if subjects were under any anti-reflux therapy such as proton pump inhibitors which are commonly used (15), and that could have suppressed the symptoms in those not reporting heartburn at the moment of the study.

Tack et al. have reported a higher frequency of epigastric pain in a subset of heartburn negative functional dyspepsia patients with pathological esophageal acid exposure (16). Epigastric pain together with nausea/vomiting, postprandial fullness or early satiation, comprise the different groups of symptoms in functional dyspepsia (1720 ). Hence, it is possible that the increased frequency of the first two symptoms in the IBS subjects compared to the controls in our study volunteers, is related to gastroesophageal reflux but we can not rule out that they may be a manifestation of a comorbidity with functional dyspepsia.

There is no convincing evidence for a common underlying pathophysiology for IBS and GERD, therefore the main purpose of the current study was to determine the relationship of heartburn and other related symptoms with IBS according to BMI, but contrary to our expectation, these associations were independent of BMI. As discussed by Alpers, whether comorbidities in IBS represent part of the clinical manifestations, are distinct from IBS and other functional gastrointestinal disorders, or if they are part of the somatization, remains to be elucidated (21). For example, a study among five hundred and fifty patients in London showed that IBS, tension headache, fibromyalgia, non ulcer dyspepsia, hyperventilation and non-cardiac chest pain were likely to overlap with one another and they confirmed that each functional disorder does not exist independently but instead from clusters (22). In contrast, using factor analyses, others have proven in different countries that IBS occurs independently of functional dyspepsia, gastroesophageal reflux and other gastrointestinal diseases, consistent with the Rome classification to separate upper and lower functional GI disorders (23). In other multivariate analyses it 
has also been indicated that comorbidities in IBS represent independent diseases. (24) Riedl et al. have suggested that an explanation and generalization of the complex clinical manifestation of IBS as a global disorder is not supported on the basis of the available data and those IBS patients with one or more somatic complains might represent separate IBS subgroups (10).

Finally, our study has some limitations. First, it is a symptom based study where pathological gastroesophageal reflux and/or motility abnormalities were not ruled out among our volunteers. Secondly, we did not investigate the presence of regurgitation, one of the main symptoms of GERD, as it is not part of the Rome II Modular Questionnaire. Third, we used the Rome II Modular Questionnaire and not the more current Rome III Questionnaire, as it is in the process of translation and validation for the Mexican population (25). However, we do not believe that using the Rome III rather than the Rome II criteria, would have modified our findings. Fourth, we did not analyze if there is a difference in the severity of heartburn and the other reflux-related symptoms between IBS and controls or if there is an association of severity with an increasing BMI. On the other hand, no information was obtained on whether they had seek medical attention for their heartburn and other related symptoms, therefore our results may not be generalized to patients with IBS. And last, in the current study, no information can be drawn about the underlying processes determining the association of IBS with heartburn and other reflux-related symptoms.

In conclusion, we have confirmed that heartburn and other reflux-related symptoms are more commonly reported by IBS than controls but these associations are independent of BMI. The underlying reasons for these relationships, demand further investigation.

\section{ACKNOWLEDGEMENT OF GRANTS/FUNDING}

This study was supported in part by the grant PAPIIT, IN-211107-2 of the Universidad Nacional Autónoma de México (UNAM).

\section{REFERENCES}

1. Zimmerman J, Hershcovici T. Bowel symptoms in non-erosive gastroesophageal reflux disease: nature, prevalence, and relation to Acid reflux. J Clin Gastroenterol. 2008; 42: 261-5.

2. Nastaskin I, Mehdikhani E, Conklin J, Park S, Pimentel M. Studying the overlap between IBS and GERD: a systematic review of the literature. Dig Dis Sci 2006; 51: 2113-20.

3. Raftopoulos Y, Papasavas P, Landreneau R, Young JC, Fowler D, Bergamaschi R. Clinical outcome of laparoscopic antireflux surgery for patients with irritable bowel syndrome. Surg Endosc 2004; 18: 655-9.

4. Fass R, Tougas G. Functional heartburn: the stimulus, the pain, and the brain. Gut 2002; 51: 885-92.
5. Clouse RE, Richter JE, Heading RC, Janssens J, Wilson JA. Functional esophageal disorders. Gut 1999; 45(Supl. II): II31-II6.

6. Choi CW, Kim GH, Song CS, Wang SG, Lee BJ, I H, et al. Is obesity associated with gastro pharyngeal reflux disease? World J Gastroenterol 2008; 14: 265-71.

7. Dore MP, Maragkoudakis E, Fraley K, Pedroni A, Tadeu V, Realdi $\mathrm{G}$, et al. Diet, lifestyle and Gender in gastro-esophageal reflux disease. Dig Dis Sci 2008; 53: 2027-32.

8. Dickman R, Feroze H, Fass R. Gastroesophageal reflux disease and irritable bowel syndrome: a common overlap syndrome. Curr Gastroenterol Rep 2006; 8: 261-5

9. Nocon M, Labenz J, Jaspersen D, Meyer-Sabellek W, Stolte M, Lind $\mathrm{T}$, et al. Association of body mass index with heartburn, regurgitation and esophagitis: results of the progression of gastroesophageal reflux disease study. J Gastroenterol Hepatol 2007; 22: 1728-31.

10. Riedl A, Schmidtmann M, Stengel A, Goebel M, Wisser AS, Klapp $\mathrm{BF}$, et al. Somatic comorbidities of irritable bowel syndrome: a systematic analysis. J Psychosom Res 2008; 64: 573-82.

11. Thompson WG. Research diagnostic questions for functional gastrointestinal disorders Rome II modular questionnaire. In: Drossman DA, Corazziari E, Talley NJ, Thompson WG, Whitehead WE, editors. Rome II: The Functional Gastrointestinal Disorders. Lawrence: Allen Press Inc; 2000. p. 670-88.

12. Schmulson M, Ortíz O, Santiago-Lomeli M, Gutiérrez-Reyes G, Gutiérrez-Ruiz MC, Robles-Díaz G, et al. Frequency of functional bowel disorders among healthy volunteers in Mexico City. Dig Dis 2006; 24: 342-7

13. James PT, Leach R, Kalamara E, Shayeghi M. The worldwide obesity epidemic. Obes Res 2001; 9 (Supl. 4): 228S-33S.

14. Schmulson M. Heartburn according to Rome II in Spanish-Mexico: gastroesophageal reflux must be ruled out. Rev Gastroenterol Mex 2009; 74: 74-6.

15. Martín-Echevarría E, Pereira Juliá A, Torralba M, Arriola Pereda G, Martín Dávila P, Mateos J, et al. Evaluación del uso de los inhibidores de la bomba de protones en un ser vicio de medicina interna. Rev Esp Enferm Dig 2008; 100: 76-81.

16. Tack J, Caenepeel P, Arts J, Lee KJ, Sifrim D, Janssens J. Prevalence of acid reflux in functional dyspepsia and its association with symptom profile. Gut 2005; 54: 1370-6.

17. Westbrook JI, Talley NJ. Empiric clustering of dyspepsia into symptom subgroups: a population-based study. Scand J Gastroenterol 2002; 37: 917-23.

18. Fischler B, Tack J, De Gucht V, Shkedy ZI, Persoons P, Broekaert D, et al. Heterogeneity of symptom pattern, psychosocial factors, and pathophysiological mechanisms in severe functional dyspepsia. Gastroenterology 2003; 124: 903-10.

19. Kwan AC, Bao TN, Chakkaphak S, Chang FY, Ke MY, Law NM, et al. Validation of Rome II criteria for functional gastrointestinal disorders by factor analysis of symptoms in Asian patient sample. J Gastroenterol Hepatol 2003; 18: 796-802.

20. Whitehead WE, Bassotti G, Palsson O, Taub E, Cook EC 3rd, Drossman DA. Factor analysis of bowel symptoms in US and Italian populations. Dig Liver Dis 2003; 35: 774-83.

21. Alpers D. Multidimensionality of symptom complexes in irritable bowel syndrome and other functional gastrointestinal disorders. J Psychosom Res 2008; 64: 567-72.

22. Nimnuan C, Rabe-Hesketh S, Wessely S, Hotopf M. How many functional somatic syndromes? J Psychosom Res 2001; 51(4): 54957.

23. Talley NJ, Holtmann G, Agréus L, Jones M. Gastrointestinal symptoms and subjects cluster into distinct upper and lower groupings in the community: a four nations study. Am J Gastroenterol 2000; 95: $1439-47$.

24. Whitehead WE, Palsson O, Jones KR. Systematic review of the comorbidity of irritable bowel syndrome with other disorders: what are the causes and implications? Gastroenterology 2002; 122: 1140-56.

25. Morgan D, Schmulson M, Cortes L, Squella F, Domínguez R, Rey E, et alF. The multinational translation and validation of the Spanish Rome III adult diagnostic questionnaire. Am J Gastroenterol 2008; 103(S1): S477-8. 